\title{
Single-Particle Pseudogap in Two-Dimensional Electron Systems
}

\author{
Shigeki OnODA * and Masatoshi IMADA \\ Institute for Solid State Physics, University of Tokyo, Roppongi, Minato-ku, Tokyo 106-8666
}

(Received )

\begin{abstract}
We investigate pseudogap phenomena in the $2 \mathrm{D}$ electron system. Based on the mode-mode

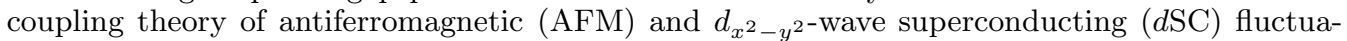
tions, single-particle dynamics is analyzed. For the parameter values of underdoped cuprates, pseudogap structure grows in the single-particle spectral weight $A(k, \omega)$ around the wave vector $(\pi, 0)$ and $(0, \pi)$ below the pseudo-spin-gap temperature $T_{\mathrm{PG}}$ signaled by the reduction of dynamical spin correlations in qualitative agreement with the experimental data. The calculated results for the overdoped cuprates also reproduce the absence of the pseudogap in the experiments. We also discuss limitations of our weak-coupling approach.
\end{abstract}

KEYWORDS: high- $T_{\mathrm{c}}$ superconductivity, pseudogap, pairing fluctuations, spin fluctuations

\section{$\S 1$. Introduction}

It is still controversial how the punusual normal state, particularly the pseudogap in the underdoped region of the high- $T_{\mathrm{c}}$ superconductors, ${ }^{\mathrm{f}}$ is understood, although various experimental studies suggest that the pseudogap develops below $T_{\mathrm{PG}}$ well above the transition temperature $T_{\mathrm{c}}$. 2. . 3 )

ARPES (angle-resolved photoemission spectra) results in the underdoped cupratest 15 suggest the followings: The quasiparticle dispersion is very flat and strongly damped around $(\pi, 0)$ and $(0, \pi)$ points ("flat spots"). The pseudogap in the single-particle excitations develops first around the flat spots below $T_{\mathrm{PG}}$, gradually extends in the direction to the $d_{x^{2}-y^{2}}$-wave gap nodes and seems to continuously merge into the $d \mathrm{SC}$ gap below $T_{\mathrm{c}}$. These experimental facts suggest that fermions in the region around the flat spots ("flat shoal region") are particularly important in considering the underdoped cuprates.

NMR experiments have revealed that the spin-lattice relaxation rate $1 /{ }^{63} T_{1} T$ has a peak around $T \sim T_{\mathrm{PG}}$, while $1 / T_{2 \mathrm{G}}$ increases down to $T_{\mathrm{c}}$, in many underdoped cuprates.6 $\mathrm{G}$. Neutron scattering experiments have clarified that the resonance peak at a finite energy $\omega^{*}$ grows below $T_{\mathrm{PG}}$, where $\omega^{*}$ is smaller for smaller doping concentrations. These results imply that though AFM fluctuations increase down to $T_{\mathrm{PG}}$, they are suppressed around $\omega=0$, while enhanced around $\omega^{*}$ below $T_{\mathrm{PG}}$.

Recently, we have studied the spin pseudogap emerging from the dominance of the $d \mathrm{SC}$ short-ranged order (SRO) over the AFM SRO by considering the AFM and $d \mathrm{SC}$ modes on an equal footing, their modemode couplings and feedback effects on the mode dampings. It has succeeded in reproducing the magnetic properties in the high- $T_{\mathrm{c}}$ cuprates. In this letter using the same parameter values as Ref.,B] we calculate the electronic spectra from the previously obtained sifceptibilities.3 Our results qualitatively reproduce and the pseudogap developing around the flat spots. 5 . The results also reproduce the flat and damped dispersion around the flat spots observed in cuprates, 40 and is reminiscent of numerical results for doped Mott insulators.1.1.2.

\section{§2. Properties of AFM and $d$ SC Fluctuations}

In the previous paper, 3 starting from the $2 \mathrm{D}$ electron system with $\mathrm{AFM}$ and $d \mathrm{SC}$ fluctuations, we have considered the effective action for AFM and $d \mathrm{SC}$ collective modes; $S=S^{(0)}+S^{(2)}+S_{\sigma \sigma}^{(4)}+S_{\mathrm{dd}}^{(4)}+S_{\sigma \mathrm{d}}^{(4)}$ with

$$
S^{(2)}=\frac{1}{T} \sum_{q}\left[\chi_{\sigma}^{-1}(q)\left|\phi_{\sigma}(q)\right|^{2}+\chi_{\mathrm{d}}^{-1}(q) \bar{\phi}_{\mathrm{d}}(q) \phi_{\mathrm{d}}(q)\right]
$$

\footnotetext{
* E-mail: onoda@ginnan.issp.u-tokyo.ac.jp
} 


$$
\begin{aligned}
S_{\sigma \sigma}^{(4)} & =\frac{u_{\sigma \sigma}}{T} \sum_{q_{1}, q_{2}, q_{3}} \phi_{\sigma}\left(q_{1}\right) \cdot \phi_{\sigma}\left(q_{2}\right) \phi_{\sigma}\left(q_{3}\right) \cdot \phi_{\sigma}\left(q_{4}\right), \\
S_{\mathrm{dd}}^{(4)} & =\frac{u_{\mathrm{dd}}}{T} \sum_{q_{1}, q_{2}, q_{3}} \bar{\phi}_{\mathrm{d}}\left(q_{1}\right) \phi_{\mathrm{d}}\left(q_{2}\right) \bar{\phi}_{\mathrm{d}}\left(q_{3}\right) \phi_{\mathrm{d}}\left(q_{4}\right), \\
S_{\sigma \mathrm{d}}^{(4)} & =\frac{2 u_{\sigma \mathrm{d}}}{T} \sum_{q_{1}, q_{2}, q_{3}} \phi_{\sigma}\left(q_{1}\right) \cdot \phi_{\sigma}\left(q_{2}\right) \bar{\phi}_{\mathrm{d}}\left(q_{3}\right) \phi_{\mathrm{d}}\left(q_{4}\right),
\end{aligned}
$$

where $\phi_{\sigma}$ and $\phi_{\mathrm{d}}\left(\bar{\phi}_{\mathrm{d}}\right)$ are the auxilary fields for spins and $d$-wave pairs, $q$ and $\sum_{q}$ represents $\left(\mathrm{i} \omega_{n}, \boldsymbol{q}\right)$ and $\sum_{n} \int \mathrm{d}^{2} \boldsymbol{q}, \omega_{n}=2 \pi n T$, and $q_{4}=-q_{1}-q_{2}-q_{3}$. Here,

$$
\begin{aligned}
& \chi_{\sigma}\left(\mathrm{i} \omega_{n}, \boldsymbol{q}\right)=A_{\sigma}\left(\xi_{\sigma}^{(0)-2}+\tilde{\boldsymbol{q}}^{2}+\frac{\gamma_{\sigma}\left|\omega_{n}\right|}{c_{\sigma}^{2}}+\frac{\omega_{n}^{2}}{c_{\sigma}^{2}}\right)^{-1}, \\
& \chi_{\mathrm{d}}\left(\mathrm{i} \omega_{n}, \boldsymbol{q}\right)=A_{\mathrm{d}}\left(\xi_{\mathrm{d}}^{(0)-2}+\boldsymbol{q}^{2}+\frac{\gamma_{\mathrm{d}}\left|\omega_{n}\right|}{c_{\mathrm{d}}^{2}}+\frac{\omega_{n}^{2}}{c_{\mathrm{d}}^{2}}\right)^{-1},
\end{aligned}
$$

are AFM and $d$ SC susceptibilities, where $\tilde{\boldsymbol{q}}=\boldsymbol{q}-\boldsymbol{Q}$ with $\boldsymbol{Q}=(\pi, \pi)$. We have neglected the phase excitations and possible long-range features of the Coulomb repulsion which may lead to gapful $d \mathrm{SC}$ excitations. It can be justified because they do not seem to alter the pseudogap structure produced by the growth of pairing amplitude. Long-range features of Coulomb repulsion which may lead to the gapful phase modes even in the $d \mathrm{SC}$ phase do not alter the following main argument. $c_{\sigma}\left(c_{\mathrm{d}}\right)$ and $\gamma_{\mathrm{d}}\left(\gamma_{\sigma}\right)$ are the velocity and the damping constant of spin (pairing) modes. The spin and the $d \mathrm{SC}$ correlation length $\xi_{\sigma}^{(0)}$ and $\xi_{\mathrm{d}}^{(0)}$ are given by $\xi_{\sigma}^{(0)}-2 \approx 1-\frac{\left|\Gamma_{\sigma}\right|}{t} \log \frac{t}{\max \left\{\mu, t^{\prime}, T\right\}} \log \frac{t}{\max \{\mu, T\}}$ and $\xi_{\mathrm{d}}^{(0)}-2 \approx 1-\frac{\left|\Gamma_{\mathrm{d}}\right|}{\sqrt{t^{2}-4 t^{\prime 2}}} \log \frac{t}{T} \log \frac{t}{\max \{\mu, T\}}$, respectively, within the Gaussian approximation for only the dominant flat-spot contributions. Using the transfers for the nearest-neighbor $t$ and the second-neighbor $t^{\prime}$ and the chemical potential $\mu$ measured from $(\pi, 0)$, the bare dispersion is given by $\varepsilon(\mathbf{k})=-2 t\left(\cos k_{x}+\cos k_{y}\right)-4 t^{\prime}\left(\cos k_{x} \cos k_{y}+1\right)-\mu$. Coupling of fermions to spins is $\Gamma_{\sigma}$ and to $d$-wave pairs is $\Gamma_{\mathrm{d}}$. We neglect the $t^{\prime}-, \mu$ - and $T$-dependences of the other parameters in (2.1) than $\xi_{\sigma, \mathrm{d}}^{(0)}-2$.

To include feedback effects from the growth of $\xi_{\sigma, \mathrm{d}}$ on $\gamma_{\sigma, \mathrm{d}}$, we take

$$
\gamma_{\sigma, \mathrm{d}}=2 \gamma_{\sigma, \mathrm{d}}^{(0)} /\left(\xi_{\sigma}^{\varphi}+\xi_{\mathrm{d}}^{\varphi}\right)
$$

from phenomenological arguments.3) If the flat-spot fermions dominate the dampings, we take $\varphi=1$. When other fermions dominate the dampings, $\varphi=0$.

The AFM-AFM, $d S C-d S C$ and AFM- $d$ SC couplings, $u_{\sigma \sigma}, u_{\mathrm{dd}}$ and $u_{\sigma \mathrm{d}}$, respectively, are assumed to be positive for cuprates, 3 where $\mathrm{AFM}$ and $d \mathrm{SC}$ fluctuations compete at low energies.

We have calculated the spin and $d \mathrm{SC}$ correlation lengths $\xi_{\sigma}$ and $\xi_{\mathrm{d}}$ by the self-consistent renormalization (SCR). Though the SCR in 2D can not describe the Kosterlitz-Thouless transition at $T=T_{\mathrm{KT}}$, we regard the transition at $T_{\mathrm{KT}} \sim T_{*}$ below which $\xi_{\mathrm{d}}$ rapidly grows and $\xi_{\sigma}$ starts decreasing.

For $\mathrm{YBa}_{2} \mathrm{Cu}_{3} \mathrm{O}_{y}$, we have estimated $t=0.25 \mathrm{eV}$ from ARPES $13,4515 c_{\sigma}=0.5 t, \gamma_{\sigma}^{(0)}=t$ and $A_{\sigma}=2 t^{-1}$ from NMR and neutron scattering experiments. 16. 17. 18, (19) For simplicity, we take $c_{\mathrm{d}}=c_{\sigma}$ and $\gamma_{\mathrm{d}}^{(0)}=\gamma_{\sigma}^{(0)}$. We take $\Gamma_{\sigma}=\Gamma_{\mathrm{d}},\left|t^{\prime}\right| \sim \mu \sim T_{\mathrm{c}}, u_{\sigma \sigma}, u_{\mathrm{dd}}$ and $u_{\sigma \mathrm{d}}$ as adjustable parameters, and choose them to give reasonable values for $T_{*} \sim T_{\mathrm{c}}, \xi_{\sigma}\left(T_{*}\right)$ and $T_{\mathrm{PG}}$ compared with observed values. We have qualitatively reproduced the overall magnetic properties observed in $\mathrm{YBa}_{2} \mathrm{Cu}_{3} \mathrm{O}_{y}$ with $y=7$ and 6.63 , for $\varphi=0$ and 1 , respectively. 3

\section{§3. Pseudogap in Single-Particle Excitations}

We show that the previously obtained $\mathrm{AFM}$ and $d \mathrm{SC}$ susceptibilities reproduce the low-energy properties of the single-particle excitations in high- $T_{\mathrm{c}}$ cuprates as well. Although we have reproduced the magnetic properties in $\mathrm{YBa}_{2} \mathrm{Cu}_{3} \mathrm{O}_{y}$, it is difficult to discuss the single-particle excitations in the same compounds, because the interpretation of the ARPES data 15,20 is still controversial due to the three-dimensionality, the contributions from $\mathrm{CuO}$ chains, lack of evidence for the $d \mathrm{SC}$ gap, and surface problems. $\mathbf{H}^{-}$Here we compare our results with ARPES data in Bi2212 with similar values for $T_{\mathrm{PG}}(\sim 170 \mathrm{~K})$ and $T_{\mathrm{c}}(\sim 83 \mathrm{~K})$ to those in $\mathrm{YBa}_{2} \mathrm{Cu}_{3} \mathrm{O}_{6.63}$.

We calculate the electronic spectra $\operatorname{Im} G(\omega, \boldsymbol{k})$ using the same parameter values as Ref.B Here, $G(\omega, \boldsymbol{k})=$ $1 /(\omega-\varepsilon(\boldsymbol{k})-\Sigma(\omega, \boldsymbol{k}))$ is the dressed Green's function. Unfortunately, there is no available formalism to 

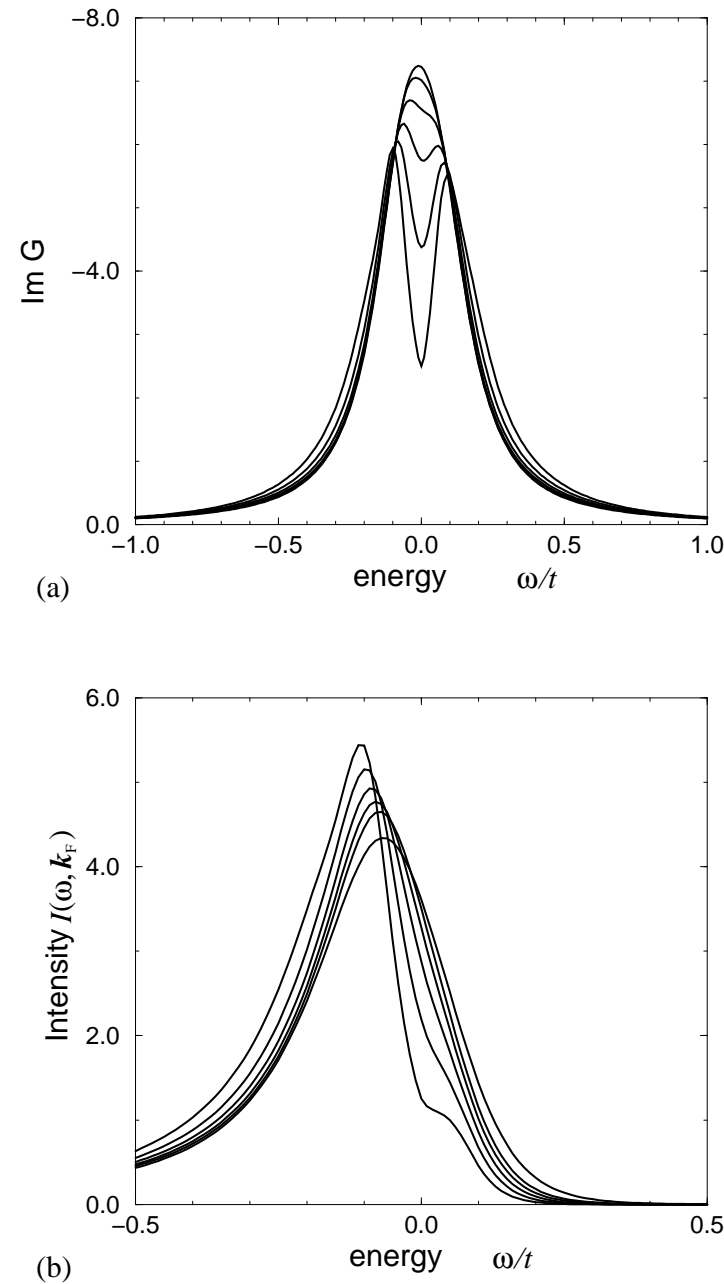

Fig. 1. Temperature variations of (a) the calculated imaginary part of the Green's function and (b) the calculated ARPES intensity for $k_{\mathrm{F}}=(\pi, 3 \pi / 64)$ for $\varphi=1$. This momentum point is on the Fermi surface and the closest to $(\pi, 0)$ in our calculation. Temperatures in the plotted data are 0.102, 0.078, 0.069, 0.06, 0.051, and 0.042 in the energy unit of $t$ from the data with larger intensity at $\omega=0$ both for (a) and (b).

reliably calculate the self-energy $\Sigma(\omega, \boldsymbol{k})$ or $G(\omega, \boldsymbol{k})$ in a self-consistent manner in the pseudogap region. A similar difficulty was already pointed out by Vilk and Tremblay in the context of the AFM fluctuations in the 2D Hubbard model.21) Here we calculate the self-energy within the 1-loop level using

$$
\begin{aligned}
& \operatorname{Im} \Sigma(\omega, \boldsymbol{k})=\int \frac{\mathrm{d}^{2} \boldsymbol{k}^{\prime}}{(2 \pi)^{2}} \int \frac{\mathrm{d} \omega^{\prime}}{2 \pi} \operatorname{Im} G^{(0)}\left(\omega^{\prime}, \boldsymbol{k}^{\prime}\right) \\
& \times\left[\Gamma_{\sigma}{ }^{2} \operatorname{Im} \chi_{\sigma}\left(\omega-\omega^{\prime}, \boldsymbol{k}-\boldsymbol{k}^{\prime}\right)\left(\operatorname{coth} \frac{\omega-\omega^{\prime}}{2 T}+\tanh \frac{\omega^{\prime}}{2 T}\right)\right. \\
& +\Gamma_{\mathrm{d}}{ }^{2} \frac{g(\boldsymbol{k})^{2}+g\left(\boldsymbol{k}^{\prime}\right)^{2}}{2} \operatorname{Im} \chi_{\mathrm{d}}\left(\omega+\omega^{\prime}, \boldsymbol{k}+\boldsymbol{k}^{\prime}\right) \\
& \left.\times\left(\operatorname{coth} \frac{\omega+\omega^{\prime}}{2 T}-\tanh \frac{\omega^{\prime}}{2 T}\right)\right],
\end{aligned}
$$

with the bare Green's function $G^{(0)}(\omega, \boldsymbol{k})$ and $g(\boldsymbol{k})=\left(\cos k_{x}-\cos k_{y}\right) / 2$. Here $\xi_{\sigma, \mathrm{d}}^{(0)}-2$ in $(2.2)$ has been replaced with $\xi_{\sigma, \mathrm{d}}^{-2}$. For the prefactor $A_{\mathrm{d}}$ in $(2.2 \mathrm{a})$, ye take $A_{\mathrm{d}}=4 t^{-1}$ to give a proper value for the midpoint shift in ARPES intensity in the pseudogap region 5 )

Figure 1(a) shows $\operatorname{Im} G\left(\omega, \boldsymbol{k}_{\mathrm{F}}\right)$ with $\boldsymbol{k}_{\mathrm{F}}$ near the flat spot at various temperatures for $\varphi=1$. For $T \geq 0.102 t$, 


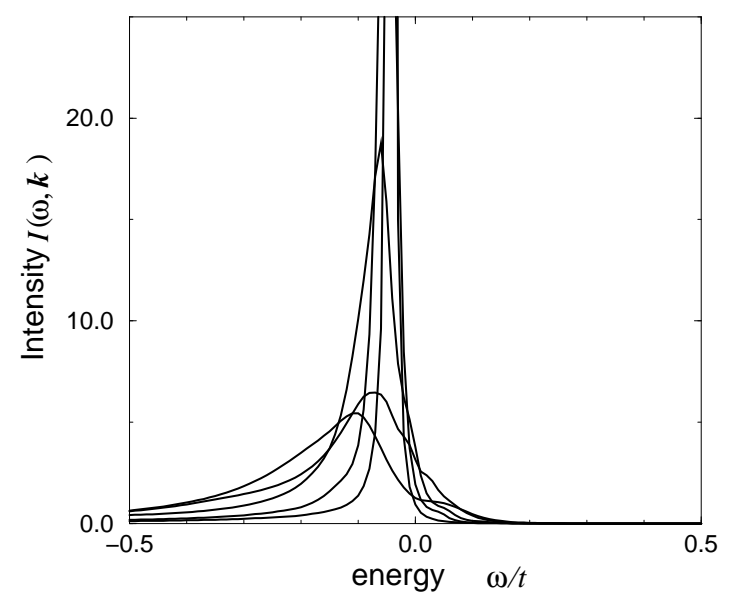

Fig. 2. Calculated ARPES intensity for various momenta $\boldsymbol{k} \sim \boldsymbol{k}_{\mathrm{F}}$ at $T=0.42 t$ in the $\varphi=1$ case. From the sharper side, $k=\left(\frac{\pi}{2}, \frac{31}{64} \pi\right),\left(\frac{40}{64} \pi, \frac{23}{64} \pi\right),\left(\frac{48}{64} \pi, \frac{15}{64} \pi\right),\left(\frac{56}{64} \pi, \frac{8}{64} \pi\right)$ and $\left(\pi, \frac{3}{64} \pi\right)$.

we have a peak at $\omega=0$, though it is damped by thermal fluctuations. At lower temperatures still above $T_{\mathrm{PG}}$, only the low-energy spectral weights gradually start decreasing. We note that low-energy fluctuations of $d$ SC-SRO grow more rapidly than those of AFM-SRO below $T_{\mathrm{PG}}(\sim 0.06 t)$ but above $T_{*}(\sim 0.02 t)$. They suppress only the low-energy part of the peak in the spectral weights. Well below $T_{\mathrm{PG}}, \operatorname{Im} G$ shows further loss of weights around $\omega=0$ and the shift to higher energies. For the same momentum $\boldsymbol{k}_{\mathrm{F}}$, we plot the intensities $I\left(\omega, \boldsymbol{k}_{\mathrm{F}}\right)=\operatorname{Im} G\left(\omega, \boldsymbol{k}_{\mathrm{F}}\right) f(\omega)$ to be observed in ARPES in Fig. 1(b), where $f$ is the Fermi function. The energy of the midpoint is nearly zero at $T=0.06 t\left(\sim T_{\mathrm{PG}}\right)$. For $T<T_{\mathrm{PG}}$, the midpoint shifts to higher binding energies. This shift amounts to $0.045 t \sim 11 \mathrm{meV}$ at $T=0.042 t(\sim 122 \mathrm{~K})$, in agreement with the experimental situations

Next we discuss the momenum dependence. We plot $I(\omega, \boldsymbol{k})$ for $\boldsymbol{k}$ 's nearest to the Fermi surface at $T=0.042 t$, in Fig. 2. It shows that the low-energy part of the single-particle excitations closer to the flat spot is under a stronger suppression, while those closer to the nodes are better understood as quasiparticles. From Fig. 2, we can see that the midpoint shift nearly vanishes at $\boldsymbol{k}=(7 \pi / 8, \pi / 8)$. For momenta closer to the gap nodes, one-peak features are recovered. These results are consistent with the ARPES data which suggest the pseudogap developing in the flat shoal region and the gradual evolution into the $d \mathrm{SC}$ gap with decrease in $T$.

Figure 3 shows the "dispersions" around the flat spot compared with the bare one. We have plotted the energy values at which $\operatorname{Im} G(\omega, k)$ has clear peaks for $T / t=0.102,0.069,0.06,0.051$ and 0.042 . At $T=0.069 t$ slightly higher than $T_{\mathrm{PG}}$, there exists only one type of "dispersion" (circles), though it jumps from one curve below the chemical potential to another above that at the remnant Fermi surface. On the other hand, for $T=0.042 t\left(<T_{\mathrm{PG}}\right)$, two types of "dispersions" appear due to the precursor effects for the $d \mathrm{SC}$ state. Besides, the dispersion in the direction from $(\pi, 0)$ to $(0,0)$ becomes flat and $\left(k_{x}-\pi\right)^{4}$-like for $\left|k_{x}-\pi\right| \delta \pi / 8$, which reminds us of numerical results in a different situation, doped antiferromagnetic insulator.11.12) We note that even fermiops distant from the remnant Fermi surface over the flat shoal region have finite spectral weights at $\omega=0.22$ It brings about some ambiguity in determining the Fermi surface around the flat spots. It also means the breakdown of the Fermi-liquid description in terms of the quasiparticles, reflecting the incoherent nature of the flat-spot fermions due to the quantum fluctuations.

We briefly mention the single-particle properties obtained for $\varphi=0$, corresponding to the cuprates in the region with no pseudogap in AFM excitations. B) In this case, our calculation on $I(\omega, k)$ also shows no clear pseudogap with a shift of midpoint energy on the Fermi surface even around the flap spots. Instead, at slightly higher temperatures than $T_{*}$, we have obtained the precursor to the $d \mathrm{SC}$ state developing in the spectral weights at finite positive energies. For underdoped $\mathrm{La}_{2-x} \mathrm{Sr}_{x} \mathrm{CuO}_{4}$, where clear spin pseudogap is not observed,23) the ARPES data24 suggest that the electrons in the diagonal direction as well as those around the flat spots have rather small weights at $\omega=0$, in contrast with other cuprates. They indicate that we have to seriously take into account the $(\pi / 2, \pi / 2)$ contributions in considering the susceptibilities for this compound. 


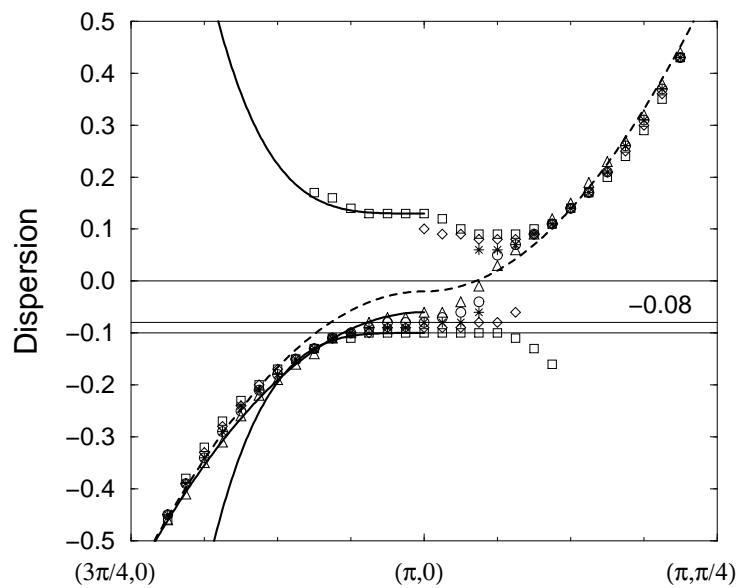

Fig. 3. Electronic dispersions scaled by $t$ at $T / t=0.102$ (triangles), 0.069 (circles), 0.06 (stars), 0.051 (diamonds) and 0.042 (squares). Solid lines fitted with triangles and squares are of the form $k_{x}^{2}$ and $k_{x}^{4}$, respectively. The dashed line represents the bare dispersion.

Our results do not reproduce the high-energy backgrounds observed in ARPES, which extend to even hundreds meV. Chubukov et al. have argued that the incoherent backgrounds and that the peak-dip-hump feature can be obtained from the coupling of fermions to overdamped spin fluctuations.25) However, spin fluctuations are underdamped in underdoped cuprates particularly below $T_{\mathrm{PG}}$. To obtain the high-energy feature of the single-particle excitations, it may be necessary to improve the formalism for calculation of the self-energy in the strong-coupling region.

\section{§4. Summary}

By using the previous solutions of the SCR which have reproduced the magnetic properties observed in high- $T_{\mathrm{c}}$ cuprates in the both regions, with and without the pseudogap 3 ) we have calculated the electronic self-energy and the Green's function.

When the mode dampings are suppressed near the transition $(\varphi=1), 1 / T_{1} T$ shows a decrease below $T_{\mathrm{PG}}\left(>T_{*}\right)$ while the spin correlation length $\xi_{\sigma}$ continues to increase until $T_{*}$. In this case, our present calculations reproduce the pseudogap behavior in single-particle excitations, and the flat and damped dispersion. Our results show that near $T_{\mathrm{PG}}$, strong $d \mathrm{SC}$ fluctuations develops an incoherent feature of single-particle excitations in the flat shoal region at lower energies than the pseudogap amplitude, from which the gaplike feature develops below $T_{\mathrm{PG}}$. The success in reproducing the pseudogap behavior in both of AFM and single-particle excitations is based on the feedback effects on the dampings of the collective modes and the competing features of low-energy AFM and $d \mathrm{SC}$ fluctuations. As concerns the former, this pseudogap formation in single-particle excitations around the flat spots qualitatively supports the selfconsistency of the phenomenological relation (2.3) used to obtain the susceptibilities, though more quantitative analyses are neededx. The latter leads to the important constraint for the mechanism of the $d$-wave superconductivity in high- $T_{\mathrm{c}}$ cuprates: The repulsion $u_{\sigma \mathrm{d}}>0$ is definitely required for reproducing the pseudogap. This means that the $d$-wave attraction is not mediated by low-energy spin fluctuations. Although it does not necessarily exclude that the attraction might come from the high-energy part of the spin fluctuations, it requires a formalism for such incoherent contributions beyond the conventional weak-coupling approach.

On the other hand, if the dampings are constant $(\varphi=0), \xi_{\sigma}$ and $1 / T_{1} T$ reach the maximum values at $T=T_{*}$. For this category, we speculate that quasiparticles around the $(\pi / 2, \pi / 2)$ point mainly contribute to the finite damping of AFM fluctuations. In this case, our present calculations show that the pseudogap region shrinks in single-particle excitations as well as in AFM excitations, and that quasiparticles around the flat spots are retained until the temperature close to $T_{\mathrm{c}}$, as in the overdoped region.

Assuming the importance of the flat-spot fermions, we have neglected fermions far from the flat spots in obtaining the susceptibilities and discussing the pseudogap formation. For more detailed discussions of the magnetic and the single-particle properties in underdoped $\mathrm{La}_{2-x} \mathrm{Sr}_{x} \mathrm{CuO}_{4}$, it is necessary to consider fermions around $(\pi / 2, \pi / 2)$ seriously. 
To go beyond the present weak-coupling approach, the direct inclusion of the self-consistent self-energy corrections in calculating the irreducible susceptibilities is required. Then the incoherence of the singleparticle excitations near the MIT may seriously modify the AFM and $d$ SC susceptibilities, especially the Curie-Weiss type form for the dynamic spin susceptibility.26)

\section{Acknowledgement}

S. O. would like to thank S. Fujiyama for discussions. The work was supported by "Research for the Future" Program from the Japan Society for the Promotion of Science under the grant number JSPS-RFTF97P01103.

[1] J. G. Bednortz and K. A. Müller : Z. Phys. B 64 (1986) 189.

[2] For a review, M. Imada, A. Fujimori and Y. Tokura: Rev. Mod. Phys. 70 (1998) 1039, Sec. IV.C.

[3] S. Onoda and M. Imada: J. Phys. Soc. Jpn., in press and cond-mat/9903030.

[4] Z.-X. Shen and D. S. Dessau: Physics Reports 253 (1995) 1; A. G. Loeser, Z.-X. Shen, D. S. Dessau, D. S. Marshall, C. H. Park, P. Fournier and A. Kapitulnik: Science 273 (1996) 325.

[5] H. Ding, T. Yokoya, J. C. Campuzano, T. Takahashi, M. Randeria, M. R. Norman, T. Mochiku, K. Kadowaki and J. Giapintzakis: Nature 382 (1996) 51; D. S. Marshall, D. S. Dessau, A. G. Loeser, C-H. Park, A. Y. Matsuura, J. N. Eckstein, I. Bozovic, P. Fournier, A. Kapitulnik, W. E. Spicer, and Z.-X. Shen: Phys. Rev. Lett. 76 (1996) 4841.

[6] H. Yasuoka, T. Imai and T. Shimizu: "Strong Correlation and Superconductivity" (Springer Verlag, Berlin, 1989 ), p.254.

[7] H. Zimmermann, M. Mali, D. Brinkmann, J. Karpinski, E. Kaldis and S. Rusiecki: Physica C 159 (1989) 681

[8] K. Ishida, Y. Kitaoka, K. Asayama, K. Kadowaki and T. Mochiku: Physica C 263 (1996) 371.

[9] H. F. Fong, B. Keimer, D. L. Milius and I. A. Aksay: Phys. Rev. Lett. 78 (1997) 713.

[10] K. Gofron, J. C. Campuzano, A. A. Abrikosov, M. Lindroos, A. Bansil, H. Ding, D. Koelling and B. Dabrowski: Phys. Rev. Lett. 73 (1994) 3302.

[11] M. Imada, F. F. Assaad, H. Tsunetsugu and Y. Motome: cond-mat/9808044 and to be published.

[12] F. F. Assaad and M. Imada: cond-mat/9811384, Eur. Phys. J. B in press.

[13] S. Massidda, J. Yu and A. J. Freeman: Phys. Lett. A 122 (1987) 198.

[14] Q. Si, Y. Zha, K. Levin and J. P. Lu: Phys. Rev. B 47 (1993) 9055.

[15] J. C. Campuzano, G. Jennings, M. Faiz, L. Beaulaigue, B. W. Veal, J. Z. Liu, A. P. Paulikas, K. Vandervoort, H. Claus, R. S. List, A. J. Arko and R. J. Barlett: Phys. Rev. Lett. 64 (1990) 2308.

[16] J. Rossat-Mignod, L. P. Renault, C. Vettier, P. Bourges, P. Burlet, J. Bossy, J. Y. Henry and G. Lapertot: Physica C 185-189 (1991) 86.

[17] S. E. Barret, D. J. Durand, C. H. Pennington, C. P. Slichter, T. A. Friedmann, J. P. Rice and D. M. Ginsberg: Phys. Rev. B 41 (1990) 6283; M. Takigawa: Phys. Rev. B 49 (1994) 4158; T. Imai, C. P. Slichter, A. P. Paulikas and B. Veal: Phys. Rev. B 47 (1993) 9158.

[18] H. Harashina, S. Shamoto, K. Kodama, M. Sato, K. Kakurai, M. Nishi, B. J. Sternlieb and G. Shirane: J. Phys. Soc. Jpn. 63 (1994) 1386.

[19] H. A. Mook, F. Doğan, and D. C. Chakoumakos: cond-mat/9811100]

[20] R. Liu, B. W. Veal, A. P. Paulikas, J. W. Downey, P. J. Kostić, S. Fleshler, U. Welp, C. G. Olson, X. Wu, A. J. Arko and J. J. Joyce: Phys. Rev. B 46 (1992) 11056.

[21] Y. M. Vilk and A.-M. S. Tremblay: J. de Phys. I 7 (1997) 1309.

[22] S. Onoda and M. Imada: unpublished.

[23] T. Imai, K. Yoshimura, T. Uemura, H. Yasuoka and K. Kosuge: J. Phys. Soc. Jpn. 59 (1990) 3846.

[24] A. Ino, C. Kim, T. Mizokawa, Z.-X. Shen, A. Fujimori, M. Takabe, K. Tamasaku, H. Eisaki and S. Uchida: J. Phys. Soc. Jpn. 68 (1999) 1496.

[25] A. V. Chubukov and J. Schmalian: cond-mat/9711041; A. V. Chubukov and D. K. Morr: cond-mat/9806200.

[26] See Ref.目) Sec.II.D,E,F and IV.C. 